\title{
Empleo de Cotrimexazol en el tratamiento de septicemias causadas por salmonellas
}

\author{
DRES.: ANTONIO BANFI ***, JOSE ROIZEN*, MARIA BEATRIZ LYNCH **, EUGENIO AMENA- \\ WAR*, EDUARDO DONOSO *. SRA, [BRIGIDA INOSTROTA *.
}

Las septicemias causadas por salmonellas representan un problema epidemiológico que aún no ha sido resuelto en nuestro país.

Desde hace 25 años, el cloramfenicol ha sido el medicamento de elección en el tratamiento de la fiebre tifoidea (1). Sin embargo, septicemias debidas a otras salmonellas, no responden al empleo de dicha droga, puesto que presentan una variable sensibilidad frente a cloramfenicol. Esto ha hecho necesario investigar otros agentes antimicrobianos que sean efectivos en tales casos y que signifiquen un menor riesgo de efectos colaterales.

Hay diversos ensayos que señalan la utilidad del cotrimexazol (Sulfametoxazol-Trimetoprim) en el tratamiento de la fiebre tifoidea $(2,3)$ pero, no hay estudios que muestren resultados para otras salmonellas de forma septicémica.

E1 presente trabajo se realizó con el objeto de evaluar la eficacia clínica y bacteriológica del Cotrimexazol en el tratamiento de septicemias producidas por salmonella typhi y otras.

Material y metodo. Este estudio se efectuó en 30 pacientes que ingresaron al Servicio de Enfermedades Infecciosas del Hospital Luis Calvo Mackenna, con diagnóstico de estado infeccioso entre los meses de Enero y Marzo de 1974.

A los 30 pacientes se les administró la droga cotrimexazol en una dosis de $40 \mathrm{mg} / \mathrm{kg}-\mathrm{p} /$ día de sulfametoxazol y $8 \mathrm{mg} / \mathrm{kg}-\mathrm{p} /$ día de trimetoprim en dos tomas diarias, luego de 48 horas sin fiebre se disminuyó a la mitad de la dosis (TABLA 1).

La temperatura se controló 6 veces al día.

Ingresaron a esta experiencia 18 hombres y 12 mujeres con una edad media de 9,3 años.

* Hospital Luis Calyo Mackenna.

* Hospital Luis Calyo Mackenna. *** Septrim $(R)$. Cedido gentilmente por el Laboratorio "SAVAL".
T A B L A 1

SEPTICEMIAS POR SALMONELLAS

Tratamiento con Cotrimexazol. Calvo Mackenna 1974

Casos: 30

SEXO: $M: 18$

Edad media: 9,3 años

F: $: 2$

Tratamiento:

Cotrimexazol:

-Sulfanetoxazol: $40 \mathrm{mg} / \mathrm{kg}-\mathrm{p} / \mathrm{d}$ ía en 2 dosis.

-Trimetoprim: $8 \mathrm{mg} / \mathrm{kg} \cdot \mathrm{p} / \mathrm{día}$ en dosis.

Luego de 48 hrs. afebriles: mitad de la dosis por 8 días afebriles.

Con el fin de pesquisar portadores se tomaron coprocultivos a los $30-60$ y 90 días después de terminado el tratamiento.

A 12 cepas de S. typhi aisladas de hemocultivo y 3 cepas de $\mathrm{S}$. typhi aisladas de orina se les estudió su sensibilidad frente a Cotrimexazol mediante el método de dilución en placa, según técnica de Steers (4).

La investigación de laboratorio en estos pacientes incluyó: hemograma - veloc. de sedimentación - orina - reacciones de aglutinación - hemocultivo y tres coprocultivos a los $30-60$ y 90 días de terminado el tratamiento.

Resultados. El diagnóstico de todos los casos estuvo fundamentado en el hemocultivo y se confirmaron 23 casos de tifoidea, 2 casos de paratifus A y 5 casos debidos a salmonella typhimurium (Tabla 2) y la serología fue positiva para el $76,6 \%$ de los casos. La sintomatología clínica observada fide semejante a la habitual en las formas septicémicas de salmonellosis (Tabla 3). 
T A B L A 2

SEPTICEMIAS POR SALMONELLAS

Tratamiento con Cotrimexazol. Calvo Mackenna 1974

Casos: 30

DIAGNOSTICO:

1.- Hemocultivo:

a) S. typhi

23

b) S. Para typhi A

c) S. typhimurium

2

5

2.-_erología:

a) Positiva

23

b) Negativa

7

T A B L A 3

SEPTICEMIAS POR SALMONELLAS

Tratamiento con Cotrimexazol. Calvo Mackenna 1974

Casos: 30

SINTOMAS Y SIGNOS:

\begin{tabular}{lcll} 
& $\%$ & & $\%$ \\
Fiebre & 100 & Roséolas & 30 \\
Lengua saburral & 80 & Vómitos & 23,3 \\
Cefalea & 63,3 & Dolor abdominal & 23,3 \\
Esplenomegalia & 46,6 & Constipación & 10 \\
Diarrea & 33,3 & & \\
\hline
\end{tabular}

T A B L A 4

SEPTICEMIAS POR SALMONELLAS

Tratamiento con Cotrimexazol. Calvo Mackenna 1974

Casos: 30

\section{I- REMISION FIEBRE}

a) Período mínimo:

b) Período máximo: Promedio:

II.- RECAIDAS :

1 caso

La remisión de la fiebre ocurrió entre un mínimo de 2 y un máximo de 8,3 días con un promedio de 5,1. Se observó la recaída en un solo caso, el cual posteriormente se trató con cloramfenicol con buen resultado (Tabla 4). La cepa de S. typhi aislada en este caso era sensible al cotrimexazol.
El resultado de la pesquisa de portadores a los 30-60 y 90 días fue negativa (Tabla 5).

El estudio de sensibilidad de las cepas de $\mathrm{S}$. typhi de hemocultivos y orina demostró actividad de la droga frente a todas ellas (Tabla 6).

Comentario. En este estudio se confirma lo señalado por distintos autores $(2,3)$, en el sentido que el cotrimexazol es una buena droga de alternativa en el tratamiento de la fiebre tifoidea. La tolerancia a la droga es muy buena y la mejoría clínica es similar a la observada con cloramfenicol.

La dosis utilizada tendría una importancia en la mejoría y en la ausencia de los portadores ya que estudios de J.S. SMITH (5) señalan que con tal dosificación se obtienen niveles bactericidas sobre salmonella typhi y con dosis menores sólo habría efecto bacteriostático.

La excelente respuesta clínica en los casos de S. typhimurium confirma lo observado in-vitro por LYNCH y col. (6) que señalan que un $98 \%$ de las cepas de $S$. typhimurium son sensibles al cotrimexazol.

En cuanto a portadores, lo que en nuestro país es un problema de importancia demostrada,

T A B L A 5

SEPTICEMIAS POR SALMONELLAS

Tratamiento con Cotrimexazol. Calvo Mackenna 1974

Casos: 30

PESQUISA PORTADORES: 30 casos

- Coprocultivos: 30 - 60 y 90 días: Negativos.

T A B L A 6

SEPTICEMIAS POR SALMONELLAS

Tratamiento con Cotrimexazol. Calvo Mackenna 1974 CIM de Cotrimexazol frente a 15 cepas de S. typhi

\begin{tabular}{cc} 
Hemocultivo & $\begin{array}{c}\text { orina } \\
\text { CIM } \mathrm{mcg} / \mathrm{ml}\end{array}$ \\
\hline $1 .-0,19$ & 0,19 \\
$2 .-0,19$ & \\
$3 .-0,39$ & \\
$4 .-0,39$ & \\
$5 .-0,39$ & 0,39 \\
$6 .-0,39$ & \\
$7 .-0,19$ & \\
$8 .-0,39$ & 0,19 \\
$9 .-0,39$ & \\
$10 .-0,39$ & \\
$11 .-0,19$ & \\
$12 .-0,19$ &
\end{tabular}


el cotrimexazol sería de utilidad, pues en el $100 \%$ de los casos del presente estudio no se observó portadores. CLEMENTI (7) ha señalado la utilidad de la droga en el control de portadores crónicos, en los cuales obtuvo un $72 \%$ de curación después de dos semanas de tratamiento.

El presente trabajo confirma la eficacia del cotrimexazol en el tratamiento de la fiebre tifoidea $(2,3)$, evidenciando por parámetros clínicos y de laboratorio y señala la posibilidad de su empleo con buenos resultados en el tratamiento de la septicemia por salmonella typhimurium.

El cotrimexazol representa una droga útil en estos casos y la disminución de la sensibilidad de la S. typhi frente a cloramfenicol en nuestro país (8) y la ausencia de portadores en los casos del presente trabajo nos hacen plantear el uso de este medicamento en el tratamiento de la fiebre tifoidea y en septicemias por salmonella typhimurium.

\section{RESUMEN}

Se estudian 30 pacientes con Septicemias por $S$. typhi, para typhi $A$ y $S$. typhimurium tratados con Cotrimexazol (Sulfametoxazol-Trimetoprim).

Se confirma el buen resultado clínico del tratamiento con dicha droga.

Se pesquisó portadores hasta 90 días de terminado el tratamiento y no se observó ningún caso.

Todas las cepas aisladas resultaron sensibles a la acción del Cotrimexazol, in vitro.

Se señala al Cotrimexazol como droga útil en el tratamiento $y$ en el control de portadores de salmonellas typhi y typhimurium.

\section{SUMMARY}

A total of 30 children with Septicemia due to Salmonellas (S. typhi - para typhi A - S. typhimurium) were treated with trimethoprim - sulfamethoxazole (cotrimexazole).

It was observed a good clinical response with that drug.

The carrier state was investigated performing stool cultures $30-60$ - 90 days after treatment was finished and no one case was observed.
All salmonellas were sensitive to the action of cotrimexazole.

The drug sulfamethoxazole - trimethoprim is very useful both in treatment and control of the carrier state of septic forms of salmonellosis.

\section{REFERENCIAS}

1.-Kraljevic, R.; Perroni, J.; Pearson, E.; Sesnic, R.; González, O.; Borel, H.; Rojas, M. y Jiménez, L. Cloromicetina y Tifoidea. Experiencia sobre 500 casos. Rev. Méd. Chil., 80: 521, 1952.

2.-Semprevivo, L.; Gamboa, R.; Silva M.; Saitúa, $M$. $T$. Treatment with bactrim of typhoid and paratyphoid fever in children. In Progress in Antimicrobial and Anticancer Chemotherapy. University Perk Press, Baltimore, I: 794-797, 1970.

3.-Snyder, M. J.; Perroni, J.; González, O.; Palomino, C.; González, C.; Music, S.; Dupont, H.; Hornick, $R$, and Woodward, T. Trimethoprim, Sulfamethoxazole in the treatment of Typhoid and Paratyphoid. Fevers. The J. of Inf. Dis. 128: 734-737, supplement, Nov. 73.

4.--Steers, E.; Foltz, E. L.; Graves, B. S. Inocula Replicating spparatus for routine testing of bacterial susceptibility. Antibiot. Chemother. (Med. Encyclopedic Inc., New York) 9: 307-311, 1959.

5.-Smith, J. S.; Discussion. The J. of Inf. Dis. 128: 747, Supplement Nov. 73.

6.-Lynch, M. B.; Valenzuela, M. C.; Donoso, E.; Cofré J. V'ilches, A. y Agüero, M. E. Estudio de sensibilidad de Enterobacterias a Sulfametoxazol, Trimetoprim y Cotrimexazol. Rev. Chil. Pediat. Presente número.

7.-Clementi, K. J. Trimethoprim - Sulfamethoxazole in the treatment of carriers of salmonella. The $J$. of Inf. Dis. 128: 738-742, Supplement, Nov. 73.

8.-Banfi, A.; Tolstov, O.; Martínez, P.; Montoya, M.; Norambuena, A.; Ocaranza, M.; Peteri, D.; Razeto, $L$. Sensibilidad de la salmonella typhi frente al cloramfenicol en los últimos 16 años. Rev. Chil. Pediat. Presente número. 